\title{
Location Coder-Decoder Android Application using Init Agent Specific Ontology for Agile Software Development
}

\author{
Sumit Kumar Mishra, V.K. Singh
}

\begin{abstract}
In the present era application based system become more popular in comparison to desktop application, Based on functionality and model, application based system categorized in two different phases: normal application \& Intelligent application . to design such application there are lot of technology avaible in the market like android, java,python and provide extra features in these application certain supported technology also available like semantic web, Multi Agent System, AOG(action On Google), Android wearable etc. In this paper author proposed a model and design application which track user information and provide support and guidelines through Agent for navigating people as well as provide a support system using mobile platform.

In this model author used semantic information features like ontology with the help of ontology based agent information tracking is more easily in compare to non supporting ontology tracker system.
\end{abstract}

Keywords: Ontology, Semantic, GPS,MAS, Agile, Scrum , Protocol.

\section{INTRODUCTION}

In past few years, social networking applications had gained a lot of support and popularity all over the world. In this model author tried to use a basic semantic web based aspects generally known as ontology. With the help of ontology author try to fetch all information and after that use this information to retrieve general co ordinates of particular location. In this application some Agent used to communicate with other agents as well as provide relevant information to the end users. In the proposed model author used 4 main key phase whose name are given below:

1. Agile_Init_Agent

2. Ontology_Agent

3. Location Finder Agent

4. Protocol specific libraries

In this application author follows agile based methodology .Agile is nothing but a simple set of some principles to provide end user satisfactory product as well as full customer satisfaction. to develop agile based project there are some methodology which follows agile rules and principles like Lean ,Scrum ,Kanban and many more. Here author follows Scrum methodology to design this application

Revised Manuscript Received on October 15, 2019.

Sumit Kumar Mishra, Department of Computer Science Engineering, Assistant Professor, Babu Banarasi Das Engineering College, Lucknow , U.P, India (Email:mishrasumit221@gmail.com)

V.K. Singh, Department of Computer Science Engineering, Professor \& Director, Babu Banarasi Das N.I.I.T. College, Lucknow,U.P, India (Email:viveksinghbbd@gmail.com)

\section{PROPOSED MODEL}

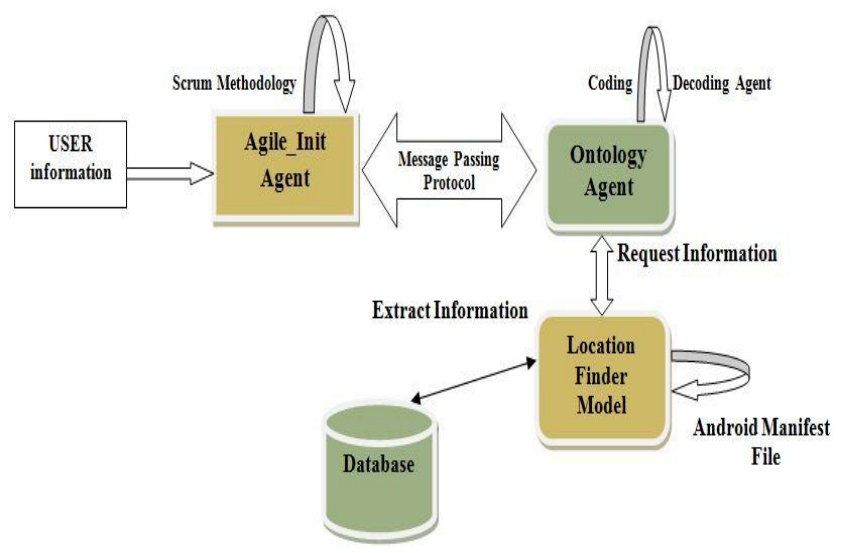

figure 1.Location Finder Information Retrieval System

\section{WORKING OF LOCATION FINDER INFORMATION MULTI AGENT APPLICATION}

In above model author internally used two different technology tried to find the location and other information. Firstly user based Agile_ Init Agent active by passing value 1.when Init Agent active then it communicate with Admin based ontology which contain all coordinates and other information related to user location.with the help of this information and previous information i.e. already stored App database location finder App take proper decision. Some Key points about the App:

- All users' information detect based on Local as well as Global ontology which is already available as form of API.

- When user successfully fill all credential \& after verified these credential By Semantic Agent then user only contact Local Agent.

- All users and devices allocated a unique Id for connecting the Agent and this Id provided by Allocator Agent .

- User can Accept or Reject Agent Request based on his/her choice.

- Voice reorganization features also available.

- $\quad$ Here to connecting user information to Agent, web semantic ontology perform most popular role.

- $\quad$ Agile_init_Agent follows scrum methodology

Published By:

Blue Eyes Intelligence Engineering 
which is the part of Agile development software,with the help of this methodology author can easily deal if requirement rapidly change .

- to provide a basic communication Agile_Init_Agent \& ontology Agent some message passing protocols follows which will establish connection between different technology and also support rapid conversion.

- Location Finder application designed Android studio so internally it follows some services that services available in manifest files.
In the above diagram author design Location based ontology using protégé tool. In this ontology author designed Init_Agent, which provide instruction to the user with the help of digital values like 1 or 0 here 1 indicate system properly activated and 0 means no agent assigned to solve user query.

For allocation and de allocation agent, another ontology designed which help to assign new agent and it also kept agent and user conversation record .

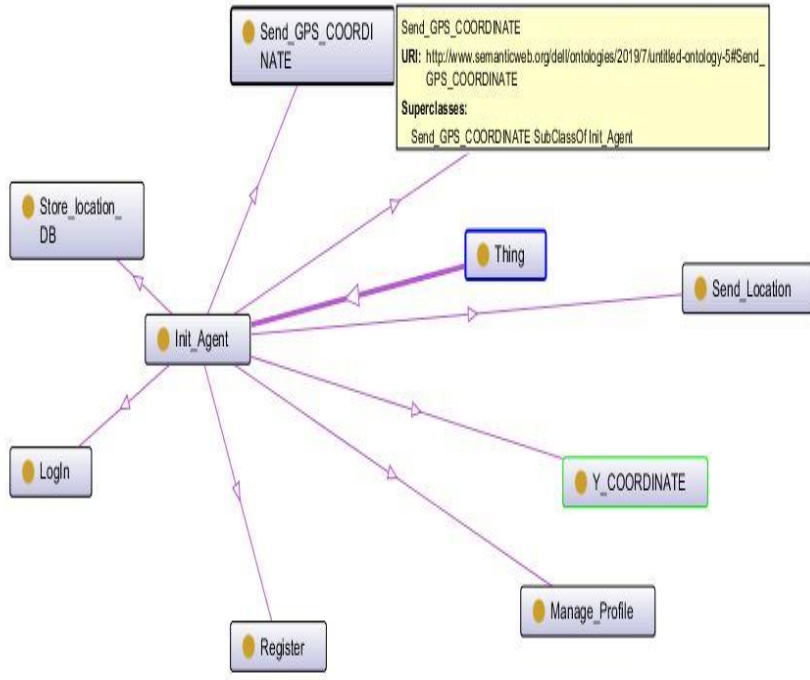

Figure 2. Ontology For location \& Coordinates detector

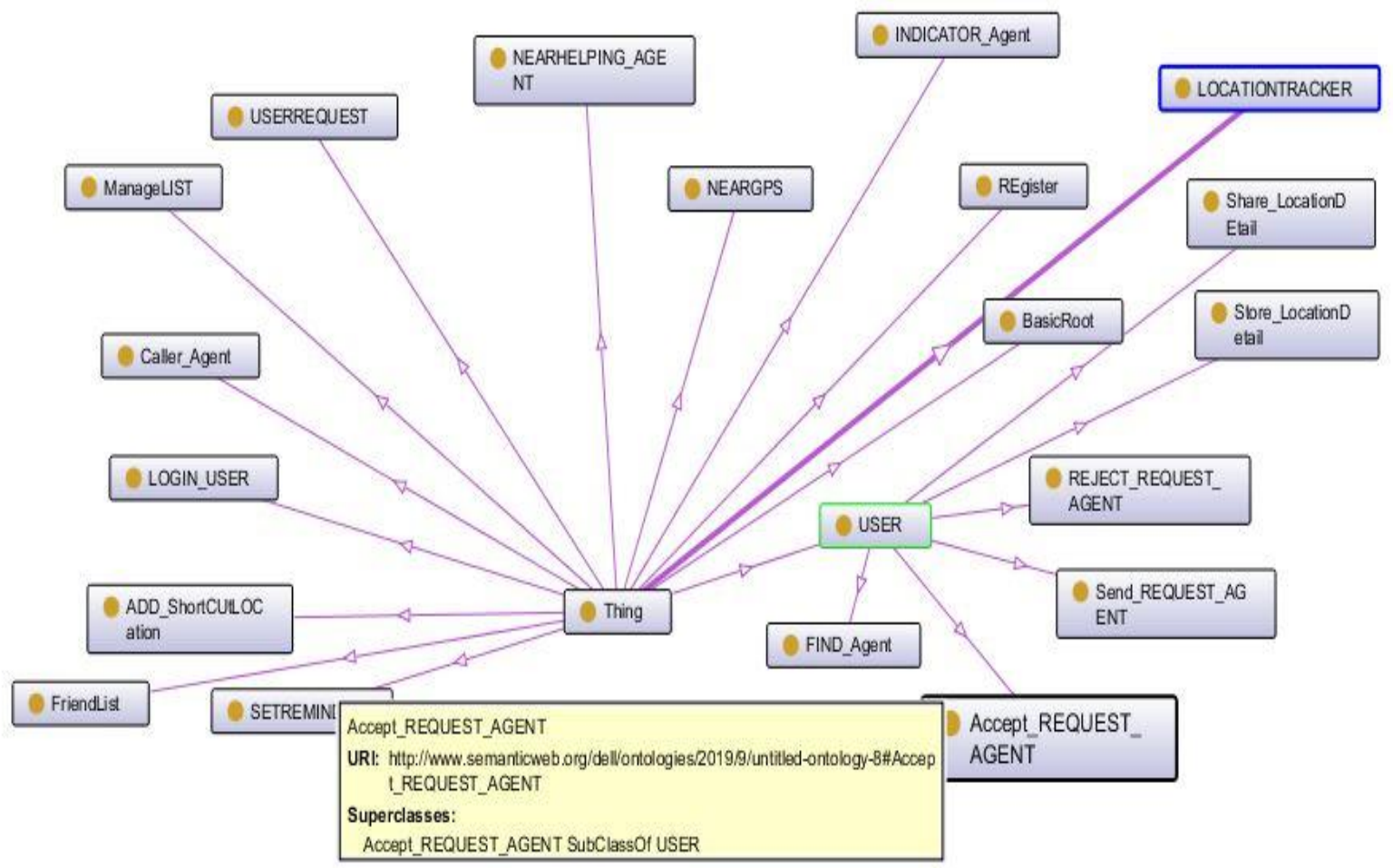

Figure 3. Ontology For Agent Allocation \& De -allocation 


\section{GPS PROVIDER AGENT}

To get access to GPS hardware of android we request using following statement Location Manager .GPS_PROVIDER \& to access GPS based information author used to semantic based ontology which is mentioned location of user which is connected via internet.

\section{CODING AND REVERSE CODING}

In the given paper author used Coding \& decoding modules which is used for translating purpose that means with the help of these we can easily translate address as well as find the latitude and longitude address. These module acts as intermediate Agents which convert Android specific calls into map based specific calls and map specific calls into android based specific routine calls.

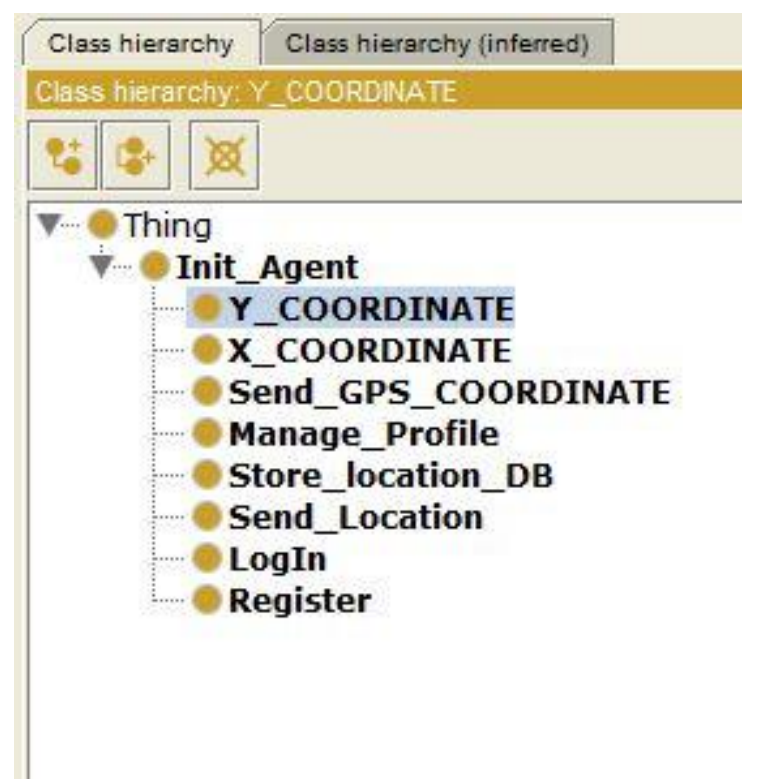

Figure 4. Class Hierarchy for ontology classes

\section{DATABASE DESIGN}

In this paper author tries to detect location as well as fetch some useful information like Local_Agent details ,History of Agent, Distance and other information for these reason some storage option required so store this information author used 2 different database (SQLite \& Firebase ) first database contain basic information like Agent_details and second contains Google_map information as well as Local address of user.

\section{DESCRIPTION OF TABLES USED} above. With the help of ontology author retrieve actual

\begin{tabular}{|c|c|c|c|}
\hline Column Name & Data Type & Allow Nulls & Descriptions \\
\hline Id & Int(11) & No & Id of user \\
\hline Name & Varchar(50) & No & Name of user \\
\hline Email & Varchar(100) & No & Email of user \\
\hline Password & Text & No & Password for user \\
\hline D.0.B. & Date & No & Date of birth \\
\hline Image & Text & Yes & Original profile image \\
\hline Thumb & Text & Yes & Compressed image \\
\hline Mobile & Varchar(12) & No & Mobile number \\
\hline Createdatetime & Datetime & No & Creation date and time \\
\hline Updatedatetime & datetime & No & Update date time \\
\hline Session & Text & Yes & Time duration spent \\
\hline Deviceid & Varchar(20) & No & Device Id id mobile \\
\hline Fcmid & Text & Yes & Firebase token id \\
\hline Actcode & Text & No & Activation code \\
\hline Active & Int(11) & No & Status of user \\
\hline Lastlogin & Datetime & No & Last login detail \\
\hline
\end{tabular}

Table 1: User Table

\section{ACTION BASED ON INIT_AGENT \& RESULTS}

\begin{tabular}{ll}
\hline & \\
\hline Send_Location & subject \\
Manage_Profile & Init_Agent \\
X_COORDINATE & Int__gent \\
Y_COORDINATE & Init_Agent \\
Send_GPS_COORDINATE & Init_Agent \\
Store_location_DB & Init_Agent \\
Login & Init_Agent \\
Register & Init_Agent
\end{tabular}

Figure 5. Action Hierarchy used by Init Agent

apart of digital values Init agent also perform several task like send location history manage user profile data, decision of coordinates as well as user basic credential. In the above figure clearly defined Init Agent action hierarchy. these action and role properly designed and integrating android studio. With the help of android studio author easily provide basic interface to the end user.

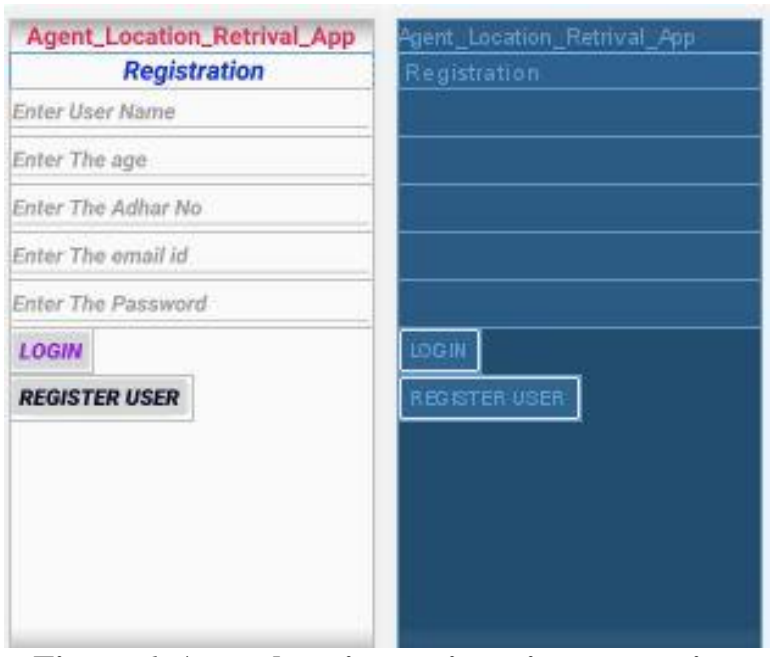

Figure 6. Agent location registration page using Android studio 


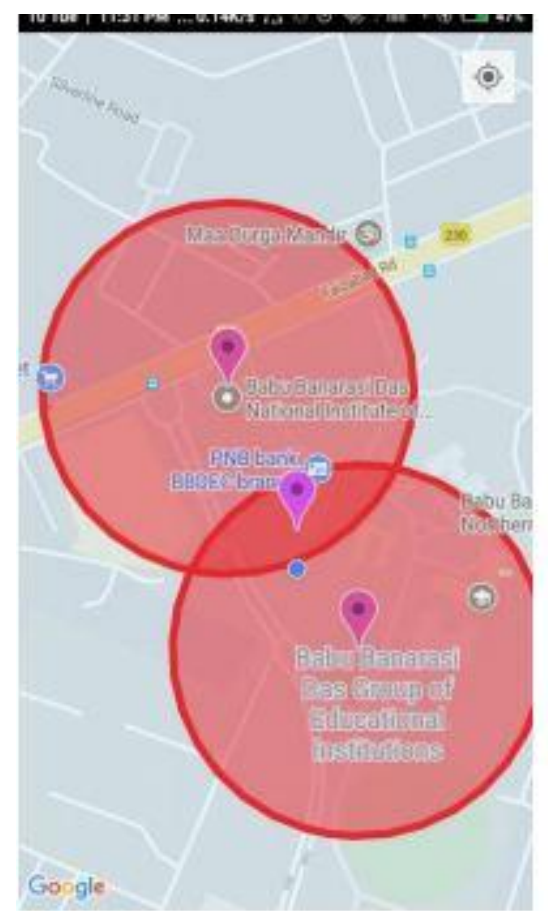

Figure 7. Agent location Detection

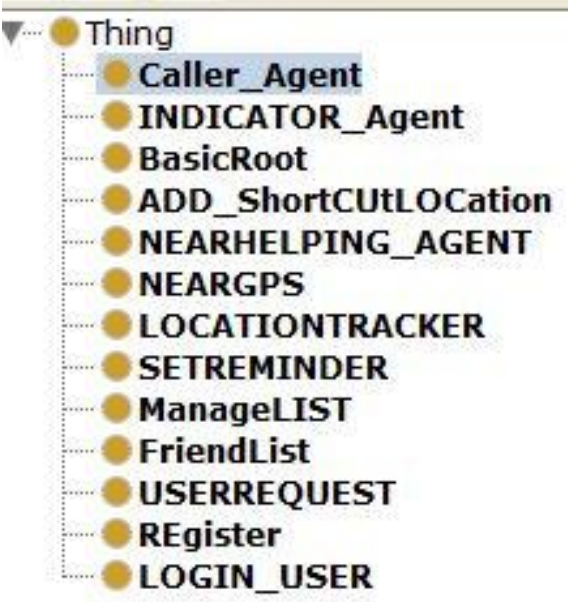

Figure 8. Location_Adder Ontology

\section{CONCLUSION}

This application track user location \& provide support system using web semantic features and follows Agile concept. this application also provide navigation direction using voice modular form \& user easily connect with agent and send request to provide guidelines them. In this paper authors are trying to established connection between android application \& ontology API using multi agent system.

\section{REFERENCES}

1. Wooldridge, Michael, Jennings, Nicholas R., Kinny, David. A methodology for agent-oriented analysis and design, AGENTS '99Proceedings of the third annual conference on Autonomous Agents(1999)

2. Sukhvir Singh, Prachi, Richa Setiya, Evaluation of Agent Oriented Software Engineering (AOSE) MethodologiesA review International Journal of Latest Research in Science and Technology Vol.1,Issue2:PageNo.94 9July.August(2012)
3. Sumit Kumar Mishra,V.K.Singh Intergrating Extended GAIA Semantic Information Retrival Model With Android Based System Using Native API International Journal of Recent Technology and Engineering (IJRTE) ISSN: 2277-3878, Volume-8 Issue-3, September 2019

4. A.Bawa S.Bhatia A REVIEW ON AGENT ORIENTED SOFTWARE ENGINEERING International Journal of Advanced Research in Computer and Communication Engineering Vol. 4, Issue 4, April 2015

5. Sumit kumar Mishra,V.K.Singh Building semantic information mediator runtime application using native API from heterogeneous data sources International Journal of Innovative Technology and Exploring Engineering (IJITEE) ISSN: 2278-3075, Volume-8 Issue-11, September 2019

6. Gaurav Kant Shankhdhar. "Introducing Two Level Verification Model for Reduction of Uncertainty of Message Exchange in Inter Agent Communication in Organizational-Multi-Agent Systems Engineering, OMaSE." IOSR Journal of Computer Engineering (IOSRJCE)IOSR Journal of Computer Engineering (IOSRJCE) 19.4 (2017): 08-18.

7. Sumit Kumar Mishra,V.K. SinghBuilding Semantic Information Retrieval System for Legal Cases from Heterogeneous Adapted and Diverse Data Sources Using Extended GAIA Methodology for Multi Agent System Proceedings - 2018 3rd International Conference On Internet of Things: Smart Innovation and Usages, IoTSIU 2018

8. Opijnen, Marc van, The European Legal Semantic Web :Completed Building Blocks and Future Work (November 22, 2012). European Legal Access Conference, November 2012.

9. Sumit Kumar Mishra,V.K. Singh Developing a multi agent system model in GAIA for court case management system as a case study and providing an extension to GAIA Proceedings - 2017 3rd International Conference on Advances in Computing, Communication and Automation (Fall), ICACCA 2017

10. Huang,W.,El-Darzi,E, Chountas,P\& Liu.,P, “Agent based Models for Community Care Systems Analysis and Design", paper in conference proceedings of the 3rd IEEE International Conference on Intelligent Systems(IEEE-IS2006),London,U.K, 2006. IEEE's Eproceedings Catalogue Number: 06EX1304C,pp273283 